\title{
Manufactura de andamios para cultivos celulares: automatización de módulos de suministro
}

\section{Manufacture of scaffolds for cell cultures: automation of supply modules}

Jorge Carvajal-Araya ${ }^{1}$

Carvajal-Araya, J. Manufactura de andamios para cultivos celulares: automatización de módulos de suministro.

Tecnología en marcha. Edición especial Movilidad Estudiantil

7. Abril, 2020. Pág.116-129.

doi) https://doi.org/10.18845/tm.v33i6.5173 


\title{
Palabras clave
}

Cultivo celular; electrohilado; nanofibras; automatización.

\section{Resumen}

Se está desarrollando un sistema de fabricación para andamios de cultivo celular. El sistema requiere crear fibras muy delgadas que están en la escala de nanómetros usando un proceso llamado electrohilado. Este artículo presenta la implementación de los módulos encargados de suministrar energía e inyectar materiales necesarios para crear nanofibras.

\section{Keywords}

Cellular culture; electrospinning; nanofibers; automation.

\begin{abstract}
A manufacturing system for cellular culture scaffolds is under development. The system requires to create very thin fibers that are in the scale of nanometers using a process called electrospinning. This article presents the implementation of the modules in charge of supplying power and injecting materials required to create nanofibers.
\end{abstract}

\section{Introducción}

El deterioro de tejidos u órganos a causa de una lesión es un problema común en el área de la salud. Tradicionalmente, este tipo de lesiones han sido asistidas con diversos tipos de tratamiento, tales como: trasplantes, intervenciones quirúrgicas, prótesis artificiales, dispositivos mecánicos, entre otros. Sin embargo, tal y como se afirma en [1], en la mayor parte de los casos el daño provocado a un tejido u órgano no puede ser reparado de forma satisfactoria.

Bajo este contexto nace el área de la ingeniería de tejidos, como una posible solución de gran potencial que consiste en la implantación de tejidos naturales, sintéticos o semi-sintéticos que son completamente funcionales y que permiten restaurar o mantener tejidos u órganos que han sufrido de alguna lesión [2]. Una de las aplicaciones más comunes es el tratamiento de quemaduras mediante el uso de piel artificial.

El Instituto Tecnológico y de Estudios Superiores de Monterrey, por medio del Centro de Innovación en Diseño y Tecnología se ha dedicado por varios años al estudio de esta área. Mediante la participación de un equipo interdisciplinario, busca el desarrollo de un sistema de manufactura de andamios híbridos para ingeniería de tejidos. El proyecto descrito en este documento se basa en la integración de los módulos de suministro de poder y de inyección del material utilizado para la confección de los andamios.

\section{Conceptos y definiciones}

La ingeniería de tejidos y medicina regenerativa consiste en un área interdisciplinaria que se encarga de la combinación de andamios, células y moléculas biológicamente activas para crear tejidos funcionales que posteriormente pueden ser utilizados para restaurar, mantener 0 mejorar tejidos dañados u órganos completos [2]. 
Según [3], el procedimiento tradicional de cultivo celular se desarrolla en un ambiente bidimensional, mediante el uso de contenedores como discos de Petri o frascos de cultivo; permitiendo el estudio del comportamiento celular en un ambiente controlado. Sin embargo, esto constituye un problema debido a que las células desarrolladas en un ambiente de dos dimensiones podrían no reaccionar de la misma manera que aquellas que se encuentran en un ambiente in vivo.

Las células que se encuentran en el interior del cuerpo humano no se limitan a un ambiente bidimensional, sino que conforman un complejo diseño en tres dimensiones conocido como matriz extracelular (ECM por sus siglas en inglés), el cual se encarga de permitir procesos como: la adhesión celular, la comunicación entre células y la diferenciación celular [3].

Para realizar procesos de cultivo celular es de gran importancia proveer un ambiente que cuente con características que se asemejen a las del entorno natural en que se desenvuelven las células de forma que estas puedan recibir los estímulos físicos y bioquímicos necesarios para su desarrollo. A estas estructuras artificiales que permiten la formación de tejidos con una estructura tridimensional se les denomina andamios. La aplicación de estos al cultivo celular permite solventar las limitaciones estructurales implicadas al realizar cultivos bidimensionales.

La fabricación de andamios tridimensionales se describe en [3] como el conjunto de tres procesos de manufactura: modelado por deposición fundida (FDM por sus siglas en inglés), electrohilado y fresado. El FDM es un método utilizado por las impresoras 3D en el que se construyen piezas capa por capa al calentar un material termoplástico hasta un estado casi líquido y colocarlo según rutas controladas por un computador [4].

El proceso de electrohilado consiste según [5] en un método simple y versátil para generar fibras ultra finas de una gran variedad de materiales como: polímeros, compuestos y cerámicas. La técnica involucra el uso de voltajes muy altos para generar un chorro del líquido que produce una fibra sólida a medida que la sustancia es estirada por la atracción estática de las cargas superficiales. La sustancia utilizada para este proyecto es preparada a partir de Policaprolactona (PCL) y acetona de grado analítico.

El desarrollo de andamios se realiza mediante deposición de capas de material intercalando las técnicas de modelado por deposición fundida y de electro-hilado. Mediante el FDM se crea una estructura de malla que posteriormente es cubierta con fibras de escala nanométrica utilizando el proceso de electro-hilado. Este proceso se repite hasta obtener la estructura deseada.

Finalmente, el proceso de fresado consiste en el uso de herramientas rotatorias utilizadas para remover material de una pieza. Este permite dar retoques finales a la estructura desarrollada.

En la figura 1 se muestra el CAD del sistema de manufactura que se pretende construir, en esta se muestra la posición de cada uno de los componentes de mayor importancia. 


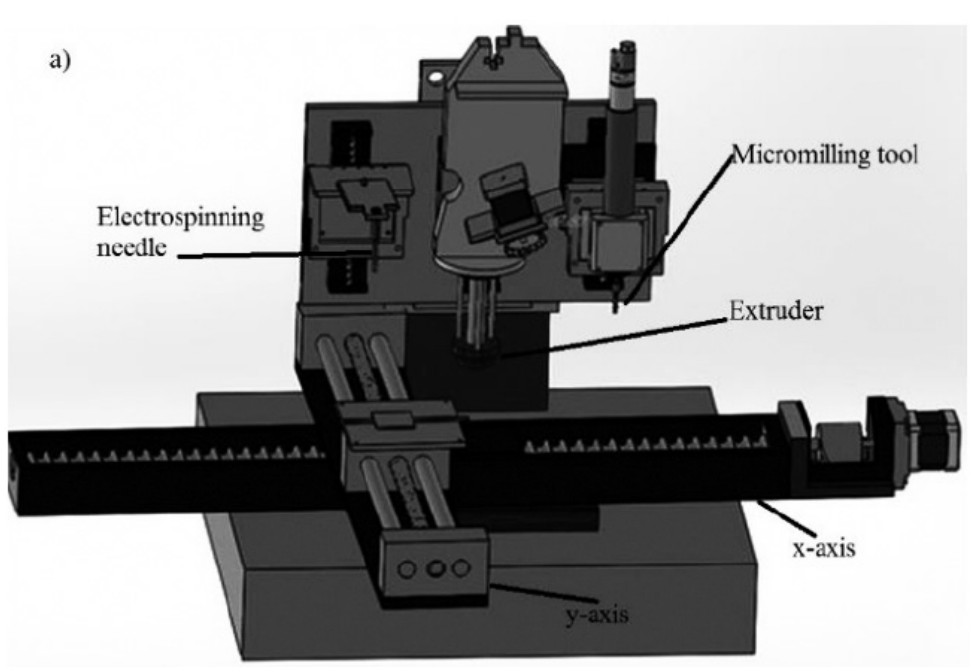

Figura 1. CAD del sistema de manufactura de andamios híbridos para ingeniería de tejidos. Tomada de [3].

\section{Estado del sistema}

A inicios del segundo semestre de 2015, era posible obtener las primeras muestras de andamios para cultivo celular, sin embargo el proceso era complicado debido a que los módulos de impresión (figura 2) y de electro-hilado (figura 3) funcionaban de forma independiente, lo cual dificultaba el desarrollo de andamios debido a que se debía llevar la muestra de un sistema al otro.

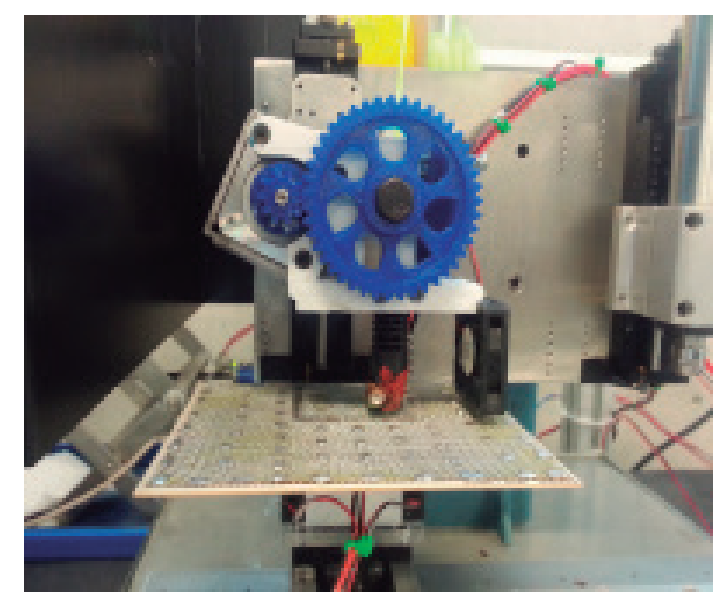

Figura 2. Sistema de impresión 


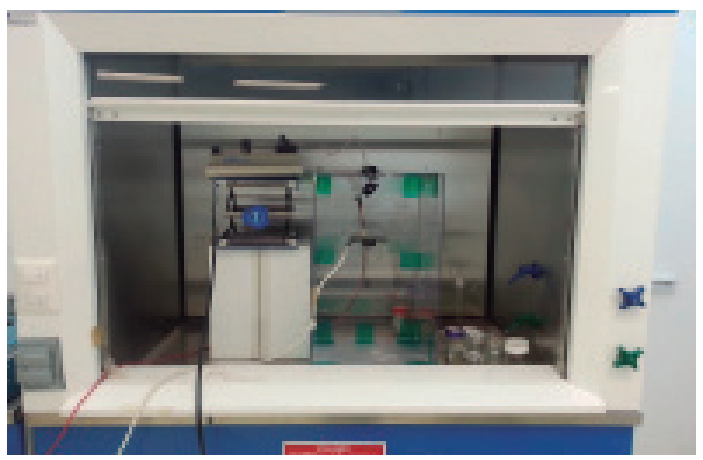

Figura 3. Sistema de electro-hilado

Para lograr que ambos sistemas trabajen de forma conjunta se deben integrar los módulos de la etapa de electro-hilado encargados de la inyección de PCL y de suministro de energía al programa principal del sistema de manufactura. Por esta razón, se pretende desarrollar una interfaz utilizando NI LabView como lenguaje de programación que permitirá controlar los módulos de forma independiente para realizar pruebas de su funcionamiento y posteriormente integrarlos al sistema desarrollado por el Dr. Christian Mendoza y el candidato a doctorado Ing. Hernán Lara.

\section{Herramientas utilizadas}

El control del sistema de manufactura se realiza mediante un computador que utiliza Windows como sistema operativo y que ejecuta un programa desarrollado con el lenguaje de programación $\mathrm{NI}$ LabView. El lenguaje de programación fue establecido por el Centro de investigación en Diseño y Tecnología (CIDyT) y conforma una limitación impuesta al proyecto. En el caso de los elementos de hardware, se utilizarán los siguientes dispositivos:

- Fuente de poder programable: Stanford Research Systems (SRS) modelo PS375.

- Bomba de desplazamiento positivo: KDScientific modelo KDS-200. 


\section{Implementación de la solución}

La implementación de la solución está dividida en tres partes: el desarrollo de la interfaz de control de la fuente de poder, el desarrollo de la interfaz que controla la fuente de desplazamiento positivo y finalmente la implementación de los módulos en el sistema de manufactura. Estas etapas serán descritas de acuerdo al orden cronológico en que fueron desarrolladas las tareas correspondientes a cada sección.

\section{Interfaz de control de la fuente de poder}

El primer paso para el desarrollo de la interfaz fue obtener los manuales correspondientes al dispositivo, los cuales pueden ser encontrados en el sitio oficial de los desarrolladores del equipo [6]. Con este documento fue posible responder a una de las principales interrogantes, el modo en que la fuente de poder se comunica con el computador. Según el manual, la forma más simple para comunicarse con la fuente es mediante el uso del puerto RS-232, mediante una serie de comandos pre-establecidos que son enviados como cadenas de caracteres.

En el documento se especifican dos tipos de comando: (1) aquellos utilizados para realizar consultas del estado del dispositivo y (2) comandos utilizados para dar órdenes al dispositivo. Para este proyecto, resulta de interés implementar una interfaz de control por lo que se dio un enfoque al segundo tipo de comandos, es decir, el utilizado para dar órdenes.

A continuación se presenta una lista de los comandos implementados, entre paréntesis se muestra la sintaxis de la instrucción, aquellos que cuentan con " $\{x\}$ ", requieren de un valor que sustituye estos caracteres:

- High Voltage On (HVOF): deshabilita el alto voltaje de la fuente.

- High Voltage On (HVON): habilita el alto voltaje de la fuente.

- Recall Configuration Zero ( $\left.{ }^{*} \mathrm{RCL} 0\right)$ : Establece las configuraciones por defecto.

- Voltage Limit (VLIM $\{x\})$ : Define el límite de voltaje al que puede operar la fuente.

- Limit Current (ILIM $\{x\})$ : Define el límite de corriente al que puede operar la fuente.

- Trip Current (ITRP $\{x\})$ : Define el límite de corriente al que puede operar la fuente y apaga el alto voltaje en caso de superar este valor

- Voltage Set (VSET $\{x\})$ : Define el valor de tensión de la fuente, pero este valor no será establecido en las terminales del equipo hasta que el alto voltaje sea activado.

Debido a que se trata de un mecanismo de comunicación bastante utilizado, National Instruments provee en su página de web un ejemplo que permite escribir y leer en el puerto serial. El código de dicho ejemplo puede ser encontrado en [7].

Este código está dividido en 4 secciones principales, las cuales se describen mediante notas en el programa mostrado en la figura 6 . Primero es necesario realizar la configuración de la comunicación serial mediante la definición de parámetros como el "baud rate", la cantidad de bits de datos, entre otros. Posteriormente se define la etapa de escritura, la cual requiere especificar la cadena de caracteres que se desea emitir. La sección de lectura es la siguiente y requiere especificar la cantidad de bits que se espera que sean leídos. Finalmente, el módulo encargado de cerrar la comunicación con el puerto. 


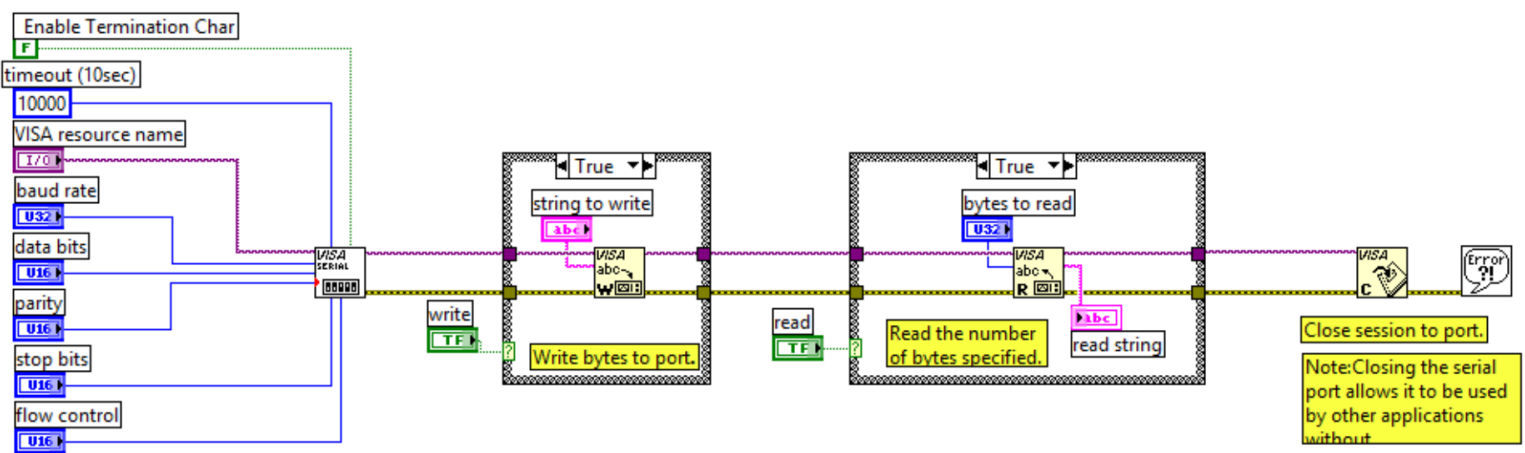

II. Onfigure Serial port paud rate lata bits, parity, stop hits and flow control)

Figura 4. Diagrama de bloques del ejemplo original provisto por la empresa National Instruments para la escritura y lectura del puerto serial.

El funcionamiento del diagrama de bloque mostrado en la figura 4 puede ser comprendido de mejor manera observando el panel frontal de dicho programa (figura 5), en el cual se especifican los parámetros de conexión en la sección izquierda, los de escritura y lectura en la sección superior izquierda.

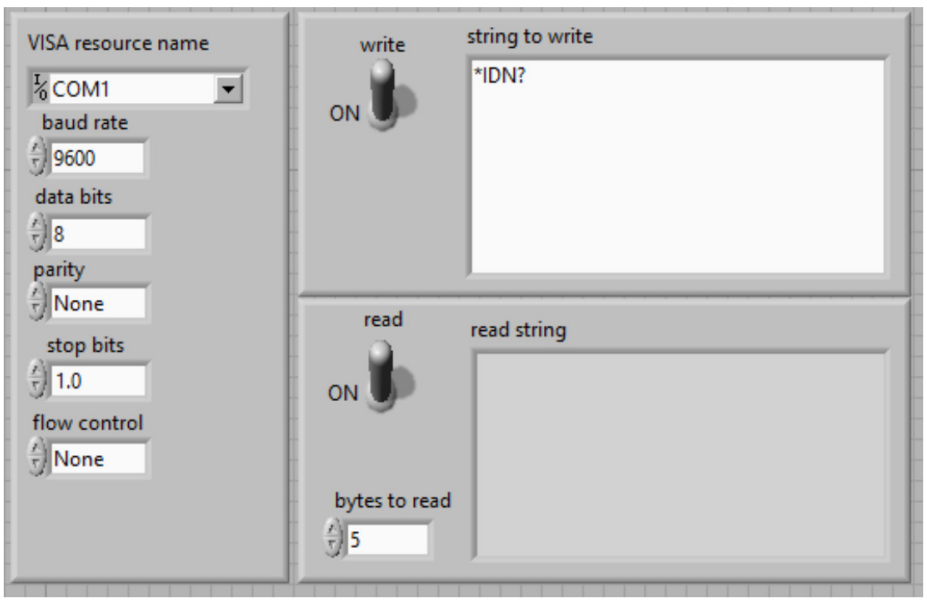

Figura 5. Panel frontal del ejemplo de escritura y lectura del puerto serial provisto por la empresa National Instruments.

Una vez que se probados los comandos que se desean utilizar con el programa descrito anteriormente, se procedió a su modificación de forma que el programa brinde funcionalidades específicas para el control de la fuente de poder. El panel frontal y diagrama de bloques del programa se muestran en las figuras 6 y 7 respectivamente.

El código se encarga de generar las cadenas de caracteres a partir de los comandos base y los valores indicados por el usuario en los campos de nivel de tensión, límite de tensión, límite de corriente, límite de disparo y los botones mostrados en la figura 6. 


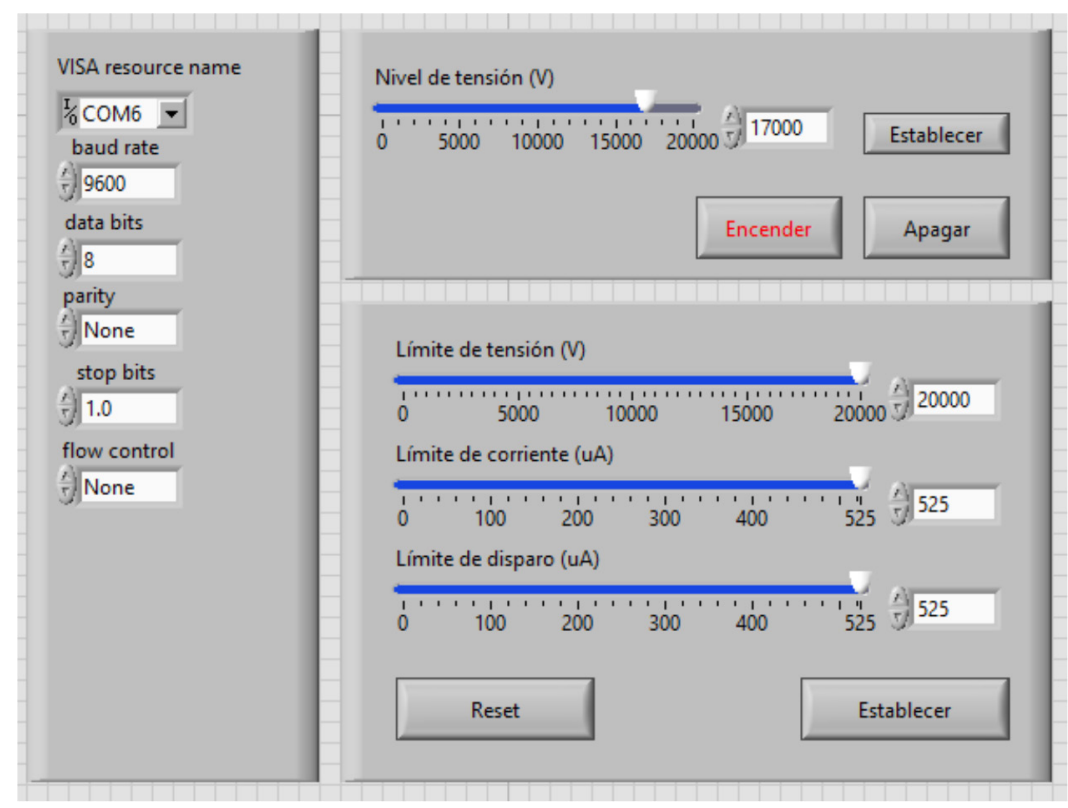

Figura 6. Panel frontal de la interfaz de control de la fuente de poder programable.

En la figura 7, se puede observar cómo se realiza el proceso de concatenación de cadenas de caracteres y estas son pasadas como parámetros a una serie de casos accionados cuando los botones se presonan.

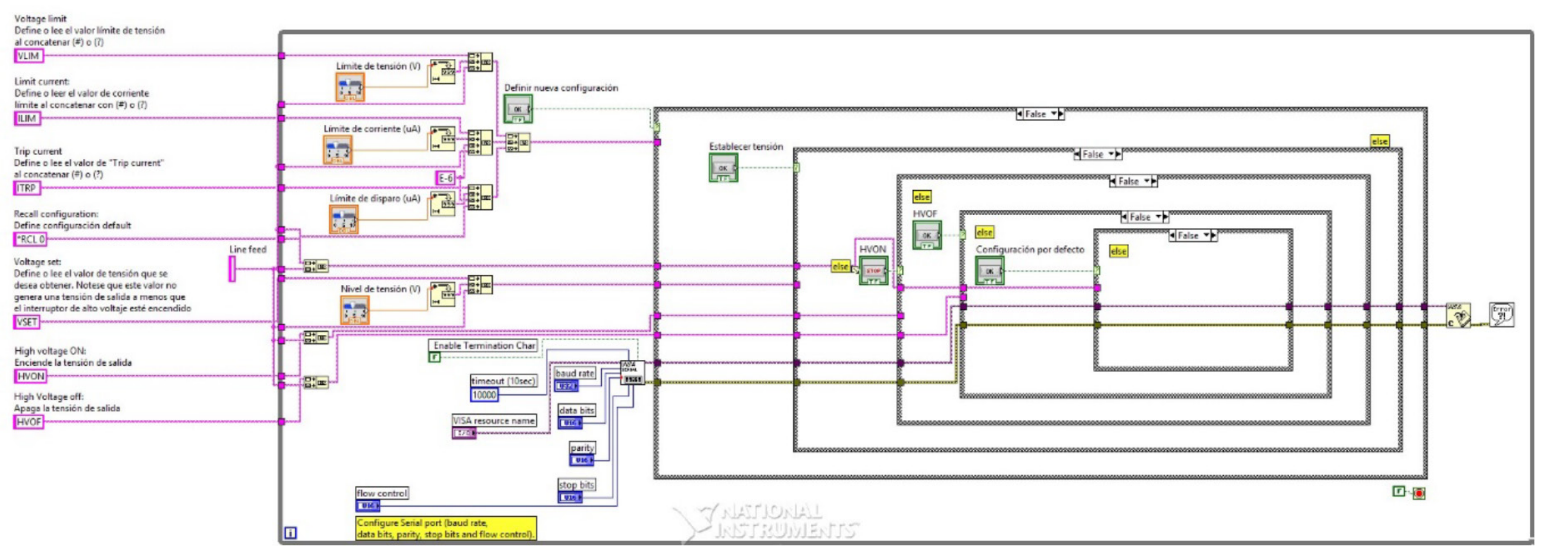

Figura 7. Diagrama de bloques de la interfaz de control de la fuente de poder programable.

\section{Interfaz de control de la bomba de desplazamiento positivo}

Al igual que con la fuente de poder, el primer paso para el desarrollo de la interfaz fue obtener los manuales correspondientes al dispositivo, los cuales pueden ser encontrados en el sitio oficial de los desarrolladores del equipo [8]. Este dispositivo cuenta con dos mecanismos de control: utilizando el protocolo RS-232 y mediante la tecnología TTL, sin embargo este último presenta una funcionalidad mucho más limitada ya que no permite el envío de comandos sino que permite realizar diferentes acciones mediante el encendido o apagado de los pines.

Se realizó un proceso de selección de los comandos que sería necesario implementar para el control de la fuente. Una vez realizada, se procedió a realizar pruebas del funcionamiento 
de estos comandos utilizando el programa de escritura y lectura del puerto serial provisto por National Instruments (el mismo utilizado para realizar las pruebas de funcionamiento de la fuente de poder).

Al realizar las pruebas se encontraron comportamientos anormales que no seguían las especificaciones del manual de usuario de la bomba de desplazamiento positivo. Tras realizar pruebas más detalladas en conjunto con el encargado del proyecto (Dr. Christian Mendoza), se determinó que el puerto serial de la bomba de desplazamiento positivo no funcionaba correctamente y por esto no se pudo realizar la implementación con el protocolo RS-232. Por esta razón se tomó la decisión de desarrollar el programa de control utilizando la tecnología TTL, a pesar de sus limitaciones.

La tecnología TTL, se basa en el encendido y apagado de ciertas líneas de control que definen funciones específicas de la bomba de desplazamiento positivo. Para esto fue necesario controlar un Arduino desde LabView para accionar las funcionalidades de la bomba, esto se realizó haciendo uso del complemento llamado Linx de LabView. De esta forma se desarrolló el programa, cuyo diagrama de bloques se muestra en la figura 8.

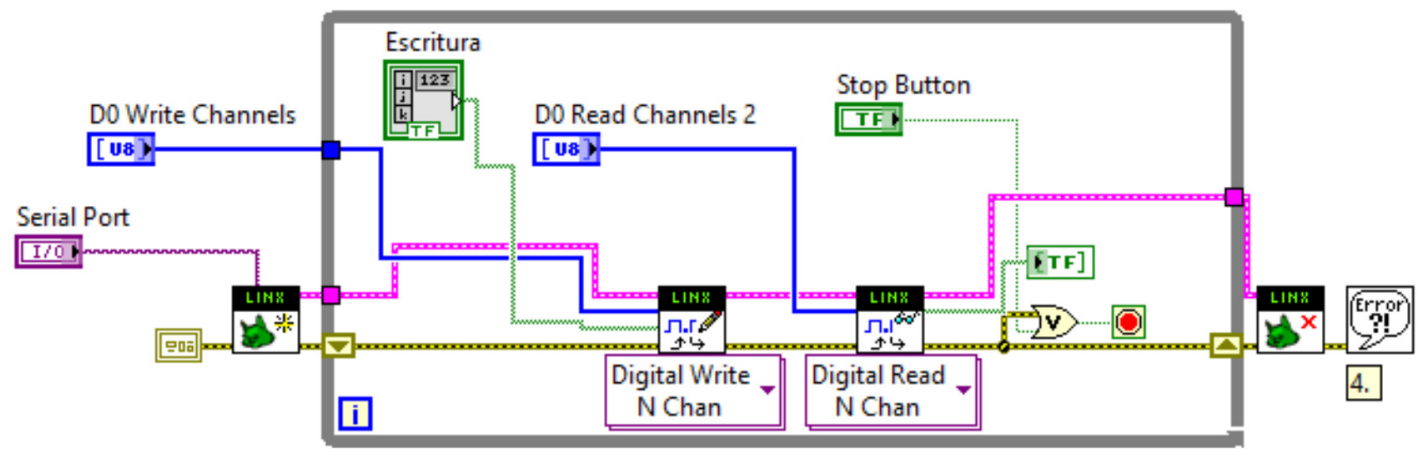

Figura 8. Programa de control de la bomba de desplazamiento positivo.

El programa tiene un flujo de trabajo muy similar al de la escritura en el puerto serial: primero, se debe realizar un proceso de inicialización en que se especifica el puerto en que el arduino se encuentra conectado. Una vez inicializado, hay un ciclo que se encarga de realizar lecturas y escrituras según la información provista por el usuario. Finalmente, existe un módulo que se encarga de cerrar la conexión.

Cabe mencionar que las únicas funcionalidades de interés que pudieron ser implementadas utilizando la tecnología TTL, son el encendido de la fuente, apagado de la fuente y lectura de algunas líneas de estatus. Todo el proceso de configuración de la misma debe realizarse previamente de forma manual ya que no es posible enviar comandos complejos a la fuente.

Las funcionalidades descritas anteriormente se pueden observar en el panel frontal del programa, el cual se presenta en la figura 9. El panel de configuración permite especificar los pines del arduino que serán utilizados para la lectura y escritura. El botón presentado al lado derecho permite cambiar el estado de encendido y apagado de la fuente y los leds de la parte baja muestran el estado de la bomba. 


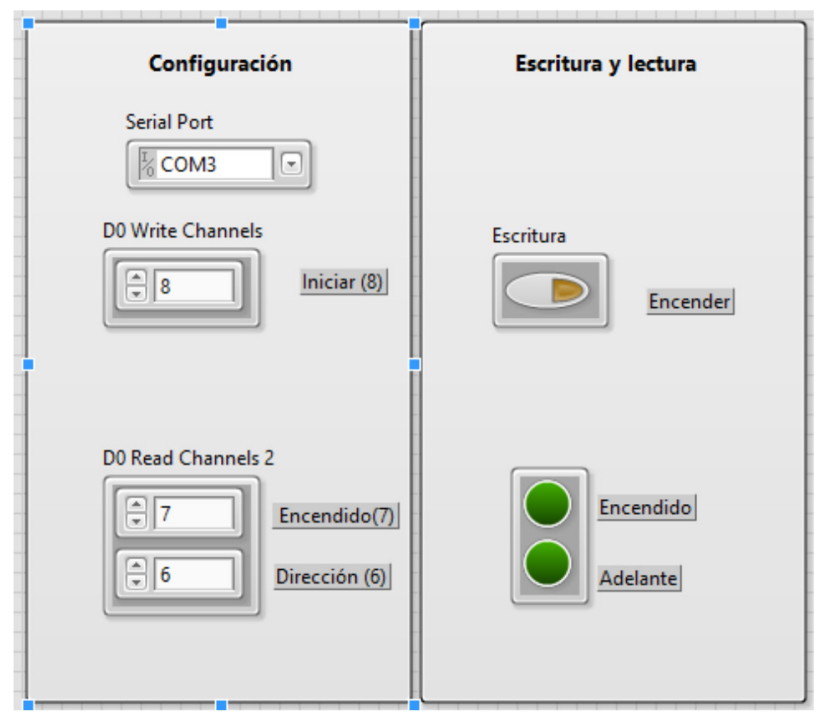

Figura 9. Panel frontal del programa de control de la bomba de desplazamiento positivo.

Las líneas de control pueden ser conectadas a cualquier pin del arduino, sin embargo para realizarlo de la forma en que se especifica en la figura 9, es necesario seguir el diagrama mostrado en la figura 10, donde se muestra claramente donde deben ser conectados cada uno de los pines.

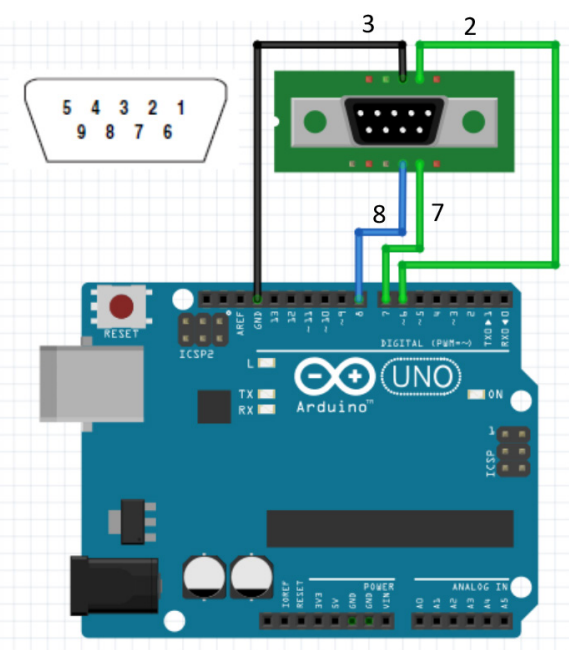

Figura 10. Diagrama de conexión.

\section{Integración al código principal}

El programa principal del sistema de manufactura fue desarrollado por el candidato a doctorado, Ing. Hernan Lara. Por razones de confidencialidad, en este documento se presenta únicamente aquellas secciones del código que se relacionan con el control de la fuente de poder y la bomba de desplazamiento positivo.

El sistema de manufactura es controlado utilizando código G, el cual consiste en un lenguaje de programación que permite indicar a una máquina que hacer y cómo lo debe hacer mediante el uso de instrucciones que indican adonde moverse, que tan rápido debe moverse y la trayectoria que esta debe seguir. Un ejemplo de los códigos utilizados puede observarse en la figura 11. 


\begin{tabular}{|c|c|}
\hline \multicolumn{2}{|c|}{; INITIALIZATION } \\
\hline G21 & ; set units to millimeters \\
\hline \multicolumn{2}{|l|}{ M107 } \\
\hline M104 S195 & ; set temperature \\
\hline G1 Z0F75 & ; lift nozzle \\
\hline G28 & ; home all axes \\
\hline M109 S195 & ; wait for temperature to be reached \\
\hline G90 & ; use absolute coordinates \\
\hline \multicolumn{2}{|l|}{ G92 E5 } \\
\hline M82 & ; use absolute distances for extrusion \\
\hline \multicolumn{2}{|c|}{; POWER SOURCE CONTROL } \\
\hline C50 & ; defines a voltage of $50 \mathrm{~V}$ \\
\hline T5 & ; waits 5 seconds \\
\hline $\mathrm{C} 80$ & ; defines voltage of $80 \mathrm{~V}$ \\
\hline T5 & ; waits 5 seconds \\
\hline B100 & ; turns on the voltage of power source \\
\hline T5 & ; waits 5 seconds \\
\hline $\mathrm{C9O}$ & ; changes the voltage while the power source is on \\
\hline T5 & ; waits 5 seconds \\
\hline B200 & ; turns off the voltage of the power source \\
\hline \multicolumn{2}{|c|}{; PUMP CONTROL } \\
\hline A100 & ; a pulse is sent tu turn the pump on \\
\hline \multicolumn{2}{|l|}{ T0.1 } \\
\hline \multicolumn{2}{|l|}{ A200 } \\
\hline T5 & ; delay \\
\hline A100 & ; a pulse is sent to turn the pump off \\
\hline \multicolumn{2}{|r|}{ r } \\
\hline \multicolumn{2}{|l|}{ A200 } \\
\hline \multicolumn{2}{|c|}{; SHUT DOWN PROCESS } \\
\hline \multicolumn{2}{|c|}{ G1 Z0 F800.000 } \\
\hline M107 & \\
\hline M104 So & ; turn off temperature \\
\hline $\mathrm{G} 28 \times 0$ & ; home $\mathrm{X}$ axis \\
\hline G28 YO & ; home $Y$ axis \\
\hline M84 & ; disable motors \\
\hline M30 & \\
\hline
\end{tabular}

Figura 11. Ejemplo de código G utilizado para el control del sistema de manufactura.

Este código es ingresado al programa de control y debe ser procesado para que se convierta en señales que determinan el comportamiento de los instrumentos que conforman el sistema de manufactura. La figura 12 muestra el panel frontal del programa encargado de controlar el sistema de manufactura y la forma en que el código es ingresado al mismo.

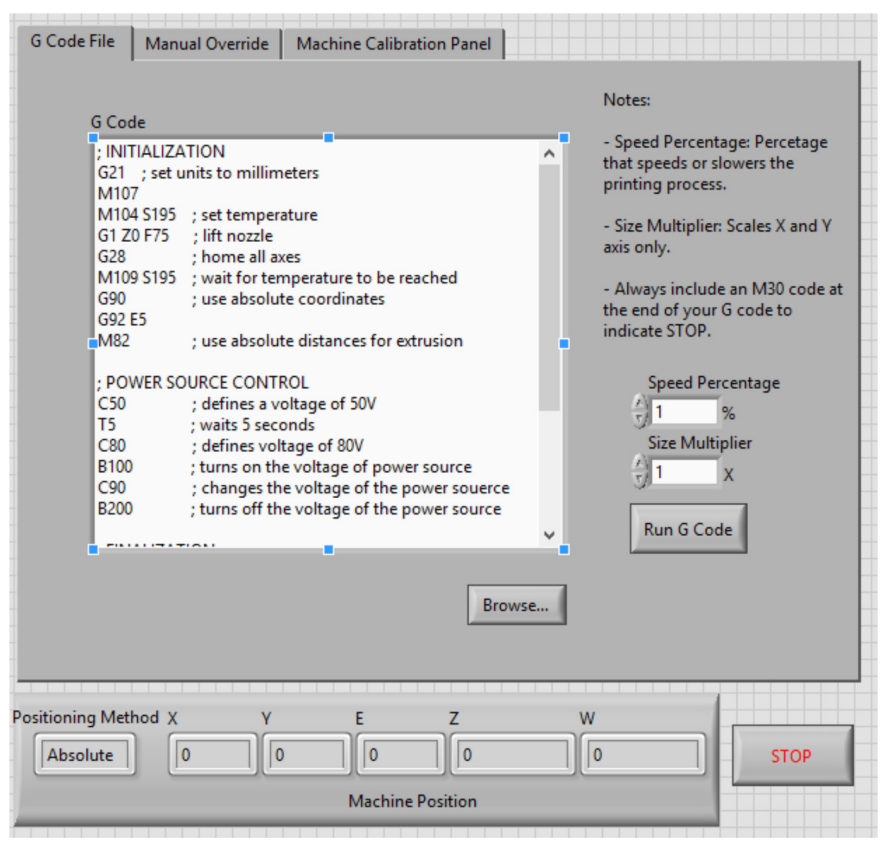

Figura 12. Panel frontal del programa principal. 
Para determinar la función que debe realizarse, el sistema utiliza comandos que están compuestos por una letra del abecedario seguida de un número. Cada letra define las diversas funciones que pueden realizar y el número que le sigue conforma un parámetro para dicha función. Por ejemplo, en el código mostrado, la "C" es utilizada para definir el valor de voltaje y el número que le sigue constituye el valor que se desea definir. En el caso de la "B", que se utiliza para encender (100) o apagar (200) la fuente según el valor que le sigue a la letra.

El conjunto de funciones que fue implementado para el sistema de manufactura es menor al que se utiliza en la interfaz de control debido a que para el programa final solo se requiere encender, apagar y cambiar el valor de voltaje de la fuente de poder. En el caso de la bomba de desplazamiento positivo, debido a las limitaciones presentadas por el fallo del puerto serial, únicamente se requiere el encendido y apagado de la misma.

Para procesar cada instrucción el sistema obtiene el primer carácter de la instrucción para determinar la función correspondiente, la cual es seleccionada mediante una estructura de "case". Las funciones para controlar la fuente de poder y la bomba son 3 y se representan mediante las letras "A", "B" y "C".

Las letras "A" y "B", se encargan del proceso de encendido/apagado de la bomba y la fuente respectivamente, mientras que la "C" permite definir la tensión que entrega la fuente de voltaje. Los códigos implementados se muestran en las figuras 13, 14 y 15. Algunos procesos de inicialización fueron realizados fuera de las estructuras presentadas en las figuras mencionadas, sin embargo, por asuntos de confidencialidad estos no serán presentados.

Como se mencionó anteriormente, al implementar la interfaz de control de la bomba de desplazamiento positivo, se utilizó la biblioteca llamada "Linx" para controlar un arduino que enciende y apaga la bomba. Sin embargo, para realizar la integración con el programa principal se debió migrar a una versión anterior de esta biblioteca llamada "Lifo". El código implementado se muestra en la figura 13.

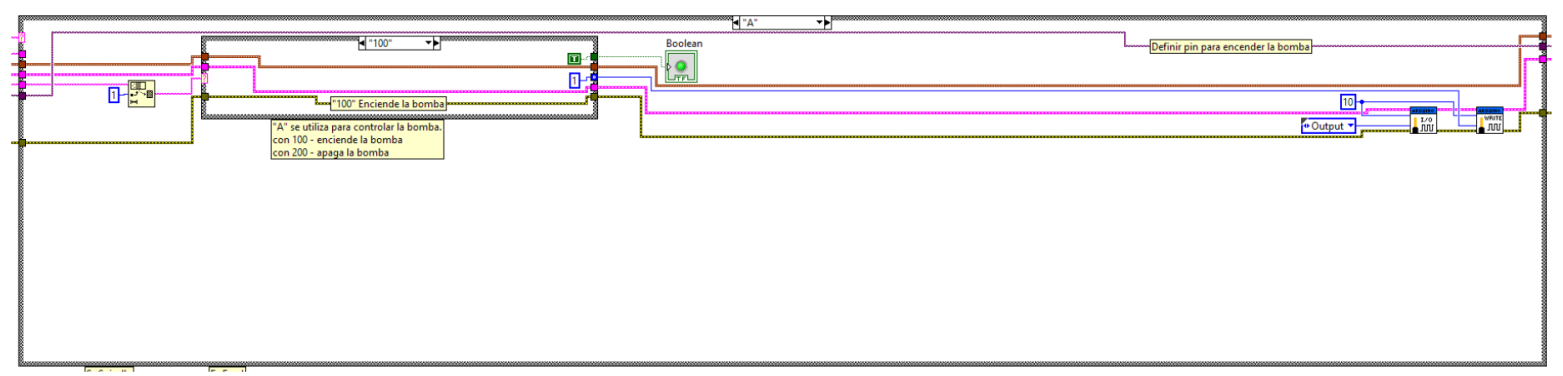

Figura 13. Caso "A": encendido y apagado de la bomba de desplazamiento positivo.

Para controlar la fuente de poder, se continúa utilizando el protocolo RS-232. En las figuras 14 y 15 se muestra el código implementado para el control de la fuente de poder. El proceso de inicialización fue realizado fuera de la estructura del case y no se presenta por razones de confidencialidad. Sin embargo, se invita al lector a revisar el código presentado en la figura 7 , donde se presenta el proceso completo. 


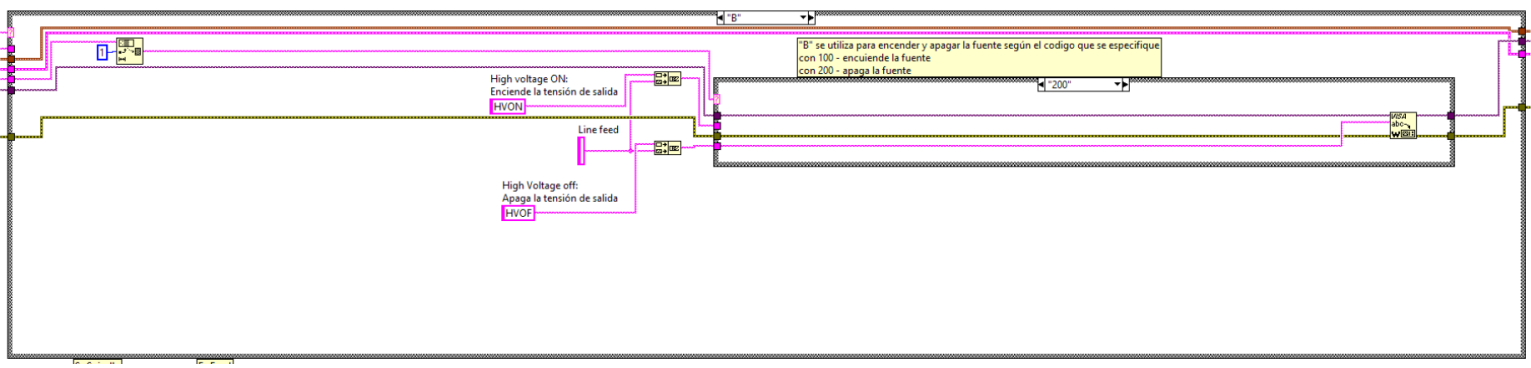

Figura 14. Caso "B": encendido y apagado de la fuente de poder.

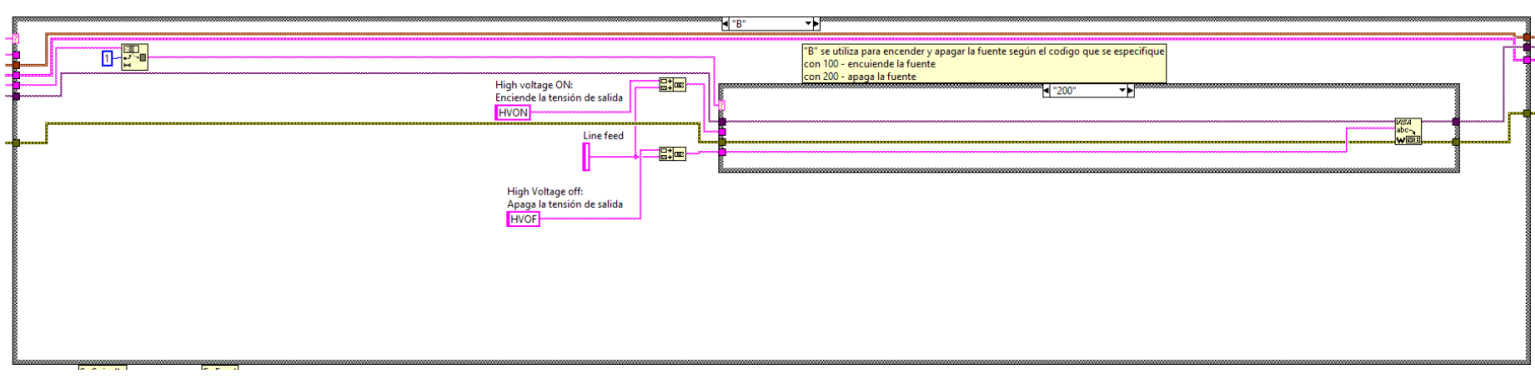

Figura 15. Caso "C": se establece nivel de tensión.

Finalmente, el código desarrollado fue presentado al Dr. Christian Mendoza una vez que fue agregado al programa principal. El código presentado en la figura 11 fue el utilizado para realizar las pruebas de todas las funcionalidades de los mecanismos implementados, con lo que se recibió el visto bueno sobre la funcionalidad del proyecto desarrollado.

\section{Discusión y resultados}

Las interfaces de control desarrolladas permitieron realizar pruebas del funcionamiento requeridas previas a la implementación en el sistema de manufactura. En el caso de la fuente de voltaje, todos los comandos presentados en las secciones fueron probados para garantizar el correcto funcionamiento de los mismos.

La bomba de desplazamiento positivo presentó algunos problemas en el puerto RS-232 por lo que se debió desarrollar una solución alternativa a la que originalmente se planeaba. A pesar de esto, la funcionalidad de mayor interés (accionar la bomba de forma automática desde un computador) fue alcanzada. La desventaja de la solución implementada es que no permite la configuración de los parámetros de flujo de la bomba, sin embargo, estos solo deben configurarse una vez.

La integración de ambos módulos se realizó de forma exitosa al código del sistema de manufactura. Durante las pruebas realizadas no fueron encontrados errores. Sin embargo, durante las mismas se determinó que existen algunos problemas estructurales que impiden que la solución pueda ser utilizada de forma inmediata.

Durante las pruebas ejecutadas se encontró que durante el desarrollo de andamios es común tener que realizar algunos ajustes manuales como la posición de la muestra o la jeringa dispensadora de PCL. Dado que el sistema se ejecuta de forma continua a partir del momento que se acciona, resulta peligroso para la persona que realiza estos ajustes el accionamiento automático de la fuente de voltaje (considerando que se trabajan con voltajes de hasta $20000 \mathrm{~V}$ ). 
A pesar de que se alcanzaron los objetivos del proyecto, los módulos no podrán ser utilizados de forma automática hasta que no se provea un mecanismo que realice los ajustes manuales y así garantizar la seguridad de los operarios.

\section{Referencias}

[1] Desconocido, «Tissue engineering, Nature Biotechnology», vol. 17, pp. 508 - 510, 2000.

[2] NIBIB, «Ingeniería de tejidos y medicina regenerativa,» julio 2013. [En línea]. Available: https://www.nibib. nih.gov/sites/default/files/Ingenier\%C3\%ADa\%20de\%20Tejidos\%20y\%20Medicina\%20Regenerativa 0.pdf. [Último acceso: 10 agosto 2015].

[3] C. Mendoza-Buenrostro, H. Lara y C. Rodriguez, «Hybrid fabrication of a 3D printed geometry embedded with PCL nanofibers for tissue engineering applications,» Procedia Engineering, pp. 128 - 134, 2015.

[4] Desconocido, «Tecnología FDM,» Stratasys, [En línea]. Available: http://www.stratasys.com/mx/impresoras-3d/ technologies/fdm-technology. [Último acceso: 10 agosto 2015].

[5] D. Li y Y. Xia, «Electrospinning of Nanofibers: Reinventing the Wheel?,» Advanced Materials, vol. 16, $n^{\circ}$ 14, p. 1151, 2004.

[6] S. R. Systems, «High Voltage Power Supplies,» [En línea]. Available: http://www.thinksrs.com/downloads/PDFs/ Manuals/PS365m.pdf. [Último acceso: 08 December 2015].

[7] N. Instruments, «Serial Communication - Basic Serial Write and Read,» National Instruments, [En línea]. Available: http://www.ni.com/example/27669/en/. [Último acceso: 08 December 2015].

[8] KDScientific, «Model 200 Series Users Manual,» [En línea]. Available: http://www.kdscientific.com/downloads/ KDS\%20Literature/Manuals/KDS Model 200 Manual.pdf. [Último acceso: 08 December 2015].

[9] ITESM, «Visión,» 13 octubre 2012. [En línea]. Available: http://www.itesm.mx/wps/wcm/connect//TESM/ Tecnologico+de+Monterrey/Nosotros/Formacion+que+Transforma+Vidas/Vision/. [Último acceso: 09 abril 2015].

[10] ITESM, «Valores,» 18 diciembre 2013. [En línea]. Available: http://www.itesm.mx/wps/wcm/connect/ITESM/ Tecnologico+de+Monterrey/Nosotros/Formacion+que+Transforma+Vidas/Valores/. [Último acceso: 09 abril 2015].

[11] ITESM, «Propósito de la institutción,» 07 mayo 2015. [En línea]. Available: http://www.itesm.mx/wps/wcm/ connect/ITESM/Tecnologico+de+Monterrey/Nosotros/Formacion+que+Transforma+Vidas/Proposito/. [Último acceso: 09 agosto 2015].

[12] CIDyT, «Centro de Innovación en Diseño y Tecnología» [En línea]. Available: http://cidyt.mty.itesm.mx/. [Último acceso: 09 agosto 2015].

[13] ITESM, «Investigación,» [En línea]. Available: http://www.itesm.mx/wps/wcm/connect/lTESM/ Tecnologico+de+Monterrey/Investigacion/. [Último acceso: 09 agosto 2015].

[14] Desconocido, «Cultura colectiva,» [En línea]. Available: http://culturacolectiva.com/wp-content/ uploads/2014/06/tecnol\%C3\%B3gico-de-monterrey.jpg. [Último acceso: 09 agosto 2015].

[15] H. Lara, C. Rodríguez y C. Mendoza, «Fuzzy inference system applied to mechanical design of bone tissue engineering scaffolds, " de Asian-Pacific Conference on Computer Aided System Engineering (APCASE), At Quito, Ecuador, 2015. 